\title{
The Study of Effect of Commercial Baicalin Combined with Cefoperazone Sulbactam Sodium on Pseudomonas aeruginosa in Vitro
}

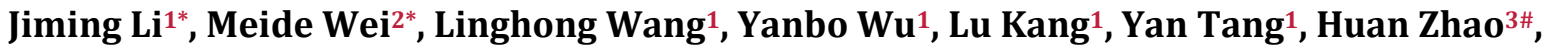 \\ Yubiao Sun ${ }^{4 \#}$
}

${ }^{1}$ Laboratory Department of the Second Affiliated Hospital of Guilin Medical College, Guilin, China; ${ }^{2}$ Laboratory Department of the Fourth Affiliated Hospital of Guangxi Medical University, Liuzhou, China; ${ }^{3}$ Laboratory Department of Chongzuo Maternal and Child Health Hospital, Chongzuo, China; ${ }^{4}$ Laboratory Department of the Second People's Hospital of Mengshan County, Mengshan County, China

Correspondence to: Huan Zhao, 122610256@qq.com; Yubiao Sun, sunyubiao12345@126.com

Keywords: Baicalin, Cefoperazone-Sulbactam Sodium, Combined Use, Pseudomonas aeruginosa, Drug Resistance, Antibacterial

Received: February 7, $2022 \quad$ Accepted: March 4, $2022 \quad$ Published: March 7, 2022

Copyright $\odot 2022$ by author(s) and Scientific Research Publishing Inc.

This work is licensed under the Creative Commons Attribution International License (CC BY 4.0).

http://creativecommons.org/licenses/by/4.0/

(c) (i) Open Access

\section{ABSTRACT}

Objective: To investigate the antibacterial effect of baicalin combined with cefoperazone-sulbactam sodium on drug-resistant strains of Pseudomonas aeruginosa. Method: Pseudomonas aeruginosa strains that are sensitive and resistant to cefoperazone-sulbactam sodium were selected to prepare different test bacterial solutions respectively; The test solutions of baicalin and cefoperazone sulbactam sodium were prepared respectively, and different test groups and control groups were set up; The drug sensitivity tests of different concentration gradients of baicalin and cefoperazone sulbactam sodium used alone and in combination were carried out for different sensitive and drug-resistant strains, and the standard strains were used as parallel control. Result: The drug susceptibility test results of the combined use of baicalin and cefoperazone-sulbactam against Pseudomonas aeruginosa drug-resistant strains were compared with the drug susceptibility results of the two used separately, and the difference was statistically significant $(P<0.05)$; The combined effect of the two was dependent on strain and concentration. Conclusion: The combined use of baicalin and cefoperazone-sulbactam sodium has a certain synergistic bacteriostatic or bactericidal effect on drug-resistant strains of Pseudomonas aeruginosa.

${ }^{*}$ Co-first author.

${ }^{\#}$ Co-corresponding author. 


\section{INTRODUCTION}

Pseudomonas aeruginosa (PAE) is a non-fermenting bacterium, belonging to gram-negative bacilli; It is widely distributed in natural environments such as air, water and soil; It is also often colonized or parasitic on gastrointestinal tract, respiratory tract, mucous membrane and skin of healthy people; When human immunity decreases, the bacterium is easy to invade human body and cause hospital infection [1]; Therefore, it is a common nosocomial opportunistic pathogen, especially in the intensive care unit (ICU) and respiratory ward. Due to the non-standard use of antibiotics and the widespread use of tumor chemotherapy and immunosuppressants in recent years, the sources of infection, infection routes and susceptible people in hospitals have increased significantly, and nosocomial infection has been gradually aggravated [2]. The incidence of infectious diseases caused by PAE is high [3] [4]. Meanwhile, the characteristics of PAE resistance, mutation and colonization become more and more complex and serious, which brings great difficulties to clinical treatment. Therefore, how to control and reduce the occurrence of nosocomial infection and seek new ways and methods to effectively treat PAE resistant strains are the common concern and urgent topic of the majority of medical workers. The purpose of this study is to find a new method of integrated traditional Chinese and Western medicine in the treatment of drug-resistant PAE, that is, to explore the antibacterial effect of baicalin combined with cefoperazone sulbactam sodium (SCF) on drug-resistant PAE, so as to provide a certain reference basis for clinical treatment. This study is as follows.

\section{MATERIALS AND METHODS}

\subsection{Materials}

1) Origin and isolation of strains the standard PAE strain (ATCC 27853) was provided by the bacteria room of the Clinical Laboratory Center of our hospital. SCF-sensitive and drug-resistant strains were isolated from sputum, blood, urine, secretions and other specimens submitted by various departments of our hospital. The drug sensitivity test was carried out by using the American BD Phoenix M50 microbial identification system; SCF sensitive strains and drug-resistant strains were determined according to the 2016 edition of American Clinical and Laboratory Standards Association [5].

2) Drugs and Reagents Baicalin extract (90.06\%): purchased from Shanxi Baoji Hongyuan Biotechnology Co., Ltd., batch No.: hy181101; SCF for injection (specification: cefoperazone $0.5 \mathrm{~g}+$ sulbactam $0.25 \mathrm{~g} /$ bottle): the manufacturer is Pfizer Pharmaceutical Co., Ltd., batch No.: x59694; M-H broth culture medium: purchased from Hangzhou Binhe microbial Reagent Co., Ltd.; Dextran standard solution: purchased from China Institute for the Control of Pharmaceutical and Biological Products.

3) Instruments Biosafety cabinet (BHC-1300IIA/B3, Jinan xinbeixi), American BD Phoenix M50 microbial identification system, self thermostatic incubator (SP), enzyme labeling instrument (Thermo Fisher multiskan FC), etc.

\subsection{Methods}

1) Isolation and identification of bacteria. Clinical samples were inoculated on MAC plates by plate streaking, and cultured at $37^{\circ} \mathrm{C}$ for $18-24 \mathrm{~h}$. The culture and isolation of PAE strains were carried out in strict accordance with the "National Clinical Inspection Procedures" [6]; and the American Clinical and Laboratory Standards Institute (CLSI)-2019 was used for identification and confirmation. At the same time, the drug sensitivity test was carried out by American BD phoenix M50 microbial identification system, and PAE sensitive and drug-resistant strains were extracted respectively.

2) Preparation of test bacterial solution [7]. An appropriate amount of PAE colonies were selected and placed in sterile $\mathrm{M}-\mathrm{H}$ broth medium, and cultured at $37^{\circ} \mathrm{C}$ for $18-24 \mathrm{~h}$. The concentration of bacterial suspension was corrected by turbidimeter, and the mother liquor with a concentration of $1 \times 108 \mathrm{CFU} / \mathrm{mL}$ was obtained; when in use, it was diluted with M-H broth medium to obtain bacterial suspension.

3) Preparation of test liquid [7]. Accurately weighed $2 \mathrm{~g}$ of commercial baicalin powder, added $5 \mathrm{ml}$ 
of sterilized purified water, added $0.1 \mathrm{M}$ hydrochloric acid, shook until dissolved, and fixed the volume to $10 \mathrm{ml}$ to obtain baicalin mother liquor $(200 \mathrm{mg} / \mathrm{ml}$ ); Dissolved $0.75 \mathrm{~g}$ of SCF powder for injection (cefoperazone $0.5 \mathrm{~g}+$ sulbactam $0.25 \mathrm{~g}$ ) with sterilized purified water to prepare $1 \mathrm{mg} / \mathrm{ml} \mathrm{SCF}$ mother liquor. The principle of drug sensitivity dilution of baicalin: the initial concentration was $1 / 2(100 \mathrm{mg} / \mathrm{ml})$, and then the drug was double-diluted with sterilized purified water to the concentration of $1 / 128(0.78125 \mathrm{mg} / \mathrm{ml})$ in the seventh tube; and the concentration range of baicalin from $1 / 128(0.78125 \mathrm{mg} / \mathrm{ml})$ to $1 / 2(100$ $\mathrm{mg} / \mathrm{ml}$ )were obtained; By diluting the SCF solution in the same way, the concentration of SCF ranges from $1 / 128(0.00390625 \mathrm{mg} / \mathrm{ml})$ to $1 / 2(0.5 \mathrm{mg} / \mathrm{L})$ were finally obtained.

4) Baicalin alone and SCF alone were used for bacterial culture [8-11]. According to the principle of NCCLS microdilution, $10 \mathrm{ml}$ sterile glass test tube was used for drug sensitivity test. Test group ( 7 concentration groups): Took 7 sterile glass test tubes, added $1 \mathrm{ml}$ of sterile purified water to each after numbering, added $1 \mathrm{ml}$ of baicalin or SCF mother liquor into tube 1, mixed well, sucked $1 \mathrm{ml}$ to tube 2, fully mixed tube 2, sucked $1 \mathrm{ml}$ to tube 3, diluted and mixed in this ratio to tube 7 , sucked $1 \mathrm{ml}$ and discarded it, added $1 \mathrm{ml}$ of $\mathrm{M}-\mathrm{H}$ broth culture medium to each tube, and then added $50 \mathrm{ml} \mu \mathrm{L}$ bacterial solution mother liquor (PAE sensitive and drug-resistant bacterial mother liquor were tested successively), and the quality control group, negative control group and positive control group were set at the same time (6 concentration groups were set in each group). Quality control group: added $1 \mathrm{ml}$ sterilized purified water and $1 \mathrm{ml}$ M-H broth medium; Negative control group: added $1 \mathrm{ml}$ baicalin or SCF and $1 \mathrm{ml} \mathrm{M}-\mathrm{H}$ broth medium; Positive control: added $1 \mathrm{ml}$ of sterilized purified water, $1 \mathrm{ml}$ of $\mathrm{M}-\mathrm{H}$ broth medium and $50 \mu \mathrm{L}$ bacterial mother liquor, cultured at $37^{\circ} \mathrm{C}$ for $20 \mathrm{~h}$.

5) Bacterial culture of baicalin combined with SCF drugs $[12,13]$. According to the principle of micro multiple dilution of NCCLS, $10 \mathrm{ml}$ sterile glass test tube was used for drug sensitivity test. Test group (7 concentration groups): took 7 sterile glass tubes, added $1 \mathrm{ml}$ sterile purified water after numbering, added $0.5 \mathrm{ml}$ baicalin and $0.5 \mathrm{ml} \mathrm{SCF}$ mother liquor into tube 1 , and the other operations were the same as the drug sensitivity test of baicalin alone or SCF alone (the drug combination test also needed the test of two bacterial mother fluids of PAE sensitive and drug resistant), and cultured at $37^{\circ} \mathrm{C}$ for 20 hours.

6) Drug sensitivity test $[14,15]$. Comply with the requirements of relevant operating procedures of automatic microbial analyzer, use the microplate reader to take the sterile medium as the blank (each test tube takes the sterile medium with the same dilution ratio as the blank) and $600 \mathrm{~nm}$ as the wavelength of light, and measure the optical density of each group at 0 hour of initial culture and 20 hours after culture at $37^{\circ} \mathrm{C}$; The effect of drugs on bacterial growth was judged by the change of optical density (that is, od value).

7) Statistical methods SPSS 20.0 statistical software was used to process the data. The test results were expressed by mean value \pm variance, and the comparison between groups was performed by one-way analysis of variance LSD-t test. The difference was statistically significant $(\mathrm{P}<0.05)$.

\section{RESULTS}

1) Drug susceptibility test results of SCF sensitive strains Baicalin alone, SCF alone, baicalin and the combination of SCF drugs had certain bacteriostatic or bactericidal effects on SCF sensitive strains (that is, there was little change in absorbance after initial culture and culture at $37^{\circ} \mathrm{C}$ for $20 \mathrm{~h}$, indicating that the bacteria were inhibited or grew slowly after culture at $37^{\circ} \mathrm{C}$ for $20 \mathrm{~h}$, and the drugs used alone or in combination had certain bacteriostatic or bactericidal effects), and their effect was related and dependent on drug concentration; there was no significant difference in the comparison of OD value of drug sensitivity test results between drug alone and drug combination after cultured at $37^{\circ} \mathrm{C}$ for 20 hours. The results of the optical density test for single and combined use of drugs at initial culture time 0 and the drug sensitivity test for sensitive strains after culture at $37^{\circ} \mathrm{C}$ for $20 \mathrm{~h}$ are shown in Table 1 below.

2) The results of drug sensitivity test of SCF resistant strains.

Baicalin alone and SCF alone had no bacteriostatic or bactericidal effect on SCF resistant strains (that is, the absorbance changed significantly after initial culture and culture at $37^{\circ} \mathrm{C}$ for $20 \mathrm{~h}$, and the OD value 
Table 1. Comparison of the absorbance of each drug and standard PAE-sensitive strains at initial culture and at $37^{\circ} \mathrm{C}$ for $20 \mathrm{~h}$.

\begin{tabular}{ccccccccc}
\hline \multirow{2}{*}{$\begin{array}{c}\text { Bacterial } \\
\text { strain }\end{array}$} & $\begin{array}{c}\text { Culture } \\
\text { time }\end{array}$ & $1 / 2$ & $1 / 4$ & $1 / 8$ & $1 / 16$ & $1 / 32$ & $1 / 64$ & $1 / 128$ \\
\cline { 3 - 8 } & $0 \mathrm{~h}$ & 0.201 & 0.182 & 0.161 & 0.126 & 0.090 & 0.061 & 0.050 \\
\multirow{2}{*}{ SCF } & $20 \mathrm{~h}$ & 0.198 & 0.178 & 0.158 & 0.120 & 0.086 & 0.053 & 0.048 \\
\hline \multirow{2}{*}{ Baicalin } & $0 \mathrm{~h}$ & 0.230 & 0.218 & 0.190 & 0.152 & 0.112 & 0.081 & 0.063 \\
& $20 \mathrm{~h}$ & 0.222 & 0.205 & 0.180 & 0.142 & 0.113 & 0.078 & 0.061 \\
\hline \multirow{2}{*}{ SCF + Baicalin } & $0 \mathrm{~h}$ & 0.212 & 0.205 & 0.182 & 0.149 & 0.119 & 0.088 & 0.060 \\
& $20 \mathrm{~h}$ & 0.208 & 0.196 & 0.179 & 0.138 & 0.103 & 0.066 & 0.052 \\
\hline
\end{tabular}

Table 2. Comparison of absorbance between drugs and standard PAE resistant strains in initial culture and $20 \mathrm{~h}$ culture at $37^{\circ} \mathrm{C}$.

\begin{tabular}{ccccccccc}
\hline \multirow{2}{*}{$\begin{array}{c}\text { Bacterial } \\
\text { strain }\end{array}$} & $\begin{array}{c}\text { Culture } \\
\text { time }\end{array}$ & \multicolumn{7}{c}{ Relative drug concentration } \\
\cline { 3 - 9 } SCF & $0 \mathrm{~h}$ & 0.203 & 0.186 & 0.165 & 0.131 & 0.093 & 0.065 & 0.048 \\
& $20 \mathrm{~h}$ & 0.652 & 0.692 & 0.755 & 0.795 & 0.836 & 0.886 & 0.950 \\
\hline \multirow{2}{*}{ Baicalin } & $0 \mathrm{~h}$ & 0.228 & 0.213 & 0.203 & 0.162 & 0.121 & 0.095 & 0.068 \\
& $20 \mathrm{~h}$ & 0.720 & 0.752 & 0.796 & 0.826 & 0.863 & 0.892 & 0.982 \\
\hline \multirow{2}{*}{ SCF + Baicalin } & $0 \mathrm{~h}$ & 0.212 & 0.201 & 0.186 & 0.158 & 0.120 & 0.086 & 0.065 \\
& $20 \mathrm{~h}$ & 0.085 & 0.096 & 0.135 & 0.186 & 0.223 & 0.228 & 0.316 \\
\hline
\end{tabular}

increased significantly, indicating that bacteria had grown after culture at $37^{\circ} \mathrm{C}$ for $20 \mathrm{~h}$, and the drug had no bacteriostatic effect or bacteriostatic effect was reduced); However, the combination of baicalin and SCF drugs had certain bacteriostatic or bactericidal effect on SCF resistant strains (that is, the absorbance changed obviously and the $\mathrm{OD}$ value decreased significantly after the initial culture and culture at $37^{\circ} \mathrm{C}$ for $20 \mathrm{~h}$, indicating that bacteria were inhibited or grew slowly after culture at $37^{\circ} \mathrm{C}$ for $20 \mathrm{~h}$, and the combined use of drugs had a certain bacteriostatic or bactericidal effect). There was also correlation and dependence between the effect and drug concentration, and the optical density of the combined drug and the single drug after $20 \mathrm{~h}$ culture at $37^{\circ} \mathrm{C}$ changed significantly, and the difference between the two was statistically significant $(\mathrm{P}<0.05)$. The optical density of the combined use and the single use of the drug at the initial incubation time of 0 hours and the drug susceptibility test results of the SCF resistant strains after incubation for 20 hours are shown in the following Table 2.

\section{DISCUSSION}

PAE is a conditional pathogen of nosocomial infection. Generally, due to the inhibition of normal flora, PAE is not pathogenic. For the PAE infection, the main antibiotics that can be selected clinically are $\beta$-Lactam antibiotics (ceftazidime, cefoperazone, aztreonam, imipenem), aminoglycoside antibiotics (such as tobramycin, amikacin, gentamicin), quinolone antibiotics (ciprofloxacin, clinfloxacin), etc. SCF is a 
compound preparation, in which sulbactam, as a broad-spectrum enzyme inhibitor, also takes into account certain antibacterial activity, and especially it has a strong and irreversible inhibitory effect on $\beta$-lactamase produced by Staphylococcus aureus and most negative bacilli, but has no activity on $\beta$-lactamase mediated by chromosomes of some negative bacilli; Among them, Cefoperazone, as a third-generation cephalosporin preparation, has relatively poor stability for $\beta$-lactamase [16]. The combined application of the two drug components will not only play a positive synergistic antibacterial activity against negative bacilli, but also its antibacterial effect is several times that of single drug [17]. Cefoperazone has bactericidal effect mainly by inhibiting the synthesis of bacterial cell wall; As an enzyme inhibitor, sulbactam can effectively protect cefoperazone from being hydrolyzed by $\beta$-lactamase which can enhance the antibacterial activity of cefoperazone [18]; Therefore, SCF is an antibacterial drugs of broad spectrum $\beta$-lactams in clinic which is commonly used in clinic and widely used in the treatment of moderate and severe bacterial infections, especially the treatment of some drug-resistant bacteria [19]. SCF also has good bacteriostatic or bactericidal effect on PAE, and the drug resistance rate is low [20]; Clinically, SCF is often used as the first choice for the treatment of conditional pathogenic bacteria and drug-resistant bacteria in nosocomial infections.

Scutellaria baicalensis is a dicotyledonous Labiatae plant; It is a Chinese herbal medicine which is often used in clinic to clear away the toxic heat; Baicalin is a flavonoid extracted and isolated from the dry root of Scutellaria baicalensis, which has the effects of antibacterial, antiviral, anti-inflammatory and anti allergic reactions, etc. Traditional Chinese medicine antibacterial therapy has the advantages of broad antibacterial spectrum and not easy to drug resistance; The application of traditional Chinese medicine antibacterial is expected to delay, inhibit or even reverse the drug resistance of PAE; Traditional Chinese medicine antibacterial therapy has the advantages of broad antibacterial spectrum and not easy to drug resistance. The application of traditional Chinese medicine antibacterial is expected to delay, inhibit or even reverse the drug resistance of PAE. The mechanisms of traditional Chinese medicine anti drug PAE mainly include: inhibiting antibacterial hydrolase, eliminating drug-resistant plasmid, restoring bacterial permeability to antibiotics, inhibiting the formation of PAE biofilm, inhibiting the efflux pump system and regulating the immune function of the body [21]. Many previous studies have found that Scutellaria baicalensis decoction can inhibit/kill Staphylococcus aureus, Escherichia coli and PAE [22, 23], and many literature [24-26] studies have shown that the antibacterial mechanism of Baicalin on PAE is to inhibit the formation of PAE biofilm and its adhesion to solids, destroy the biofilm structure, improve the sensitivity of bacteria to drugs and improve the antibacterial effect of the body. However, the antibacterial effect of baicalin is also insufficient as well as in most Chinese herbal medicines; The main reason is that the content of effective components in Chinese herbal medicine is low, and the blood drug concentration is difficult to achieve; Compared with existing antibiotics, the effectiveness of effective components is often low, which results in relatively limited antibacterial effect [27].

Over the years, due to the unreasonable use of antibiotics, the emergence and spread of multidrug-resistant bacteria have gradually increased, and the resistance rate of PAE to antibiotics, especially carbapenems, has also increased. According to the investigation of China Antibiotic Monitoring Network Center in 2017, the resistance of PAE to SCF was 24.8\% [28]; It has also been reported that PAE is resistant to a variety of antibiotics or even almost all antibiotics, which brings great challenges to the treatment of PAE induced infection and makes it a so-called "superbug" [29]. The drug resistance mechanisms of PAE mainly include: gene mutation changes channel protein and outer membrane permeability, strain enzymes change the active structure of antibiotics, gene mutations or coding enzymes change drug action target, overexpression of efflux pump-related genes, biofilm formation, integron action, etc. [30]. The increasingly serious drug resistance of PAE and the relatively limited antibacterial effect of Chinese herbal medicine have prompted people to consider the combined use of the two to achieve complementary advantages. Xu Haiying et al. found that Shuanghuanglian oral liquid combined with gentamicin showed additive effect on the drug susceptibility results of Staphylococcus aureus and PAE [31]; The study by Wang Linjing et al. found that Scutellaria baicalensis, Honeysuckle and Prunella vulgaris can antagonize ciprofloxacin on PAE by promoting the expression of efflux pump genes, and Andrographis paniculata and Galla japonica can 
also induce gentamicin to exhibit antagonizing effect on PAE [32], indicating that the combined application of traditional Chinese and western medicine has obvious synergistic antibacterial effect on PAE. The results of this study show that SCF or baicalin have no antibacterial/bactericidal effect on SCF resistant strains, but the combined use of SCF and baicalin has a certain antibacterial/bactericidal effect on SCF resistant strains, and the effect is related and dependent on drug concentration, although the mechanism of combined use of drugs on SCF resistant PAE is not clear, However, the synergistic antibacterial effect of traditional Chinese medicine and antibiotics, and the sensitivity of drug-resistant strains to corresponding antibiotics can be restored after treatment with traditional Chinese medicine, indicating that traditional Chinese medicine has the ability to reverse the resistance of drug-resistant bacteria to some extent. The mechanism of reversing bacterial resistance may be to eliminate drug-resistant plasmids, inhibit the expression of drug-resistant related genes, reduce the activity of drug-resistant related enzymes, inhibit biofilm synthesis and so on. It is precisely because baicalin may have the ability to reverse the resistance of PAE resistant strains to some extent, so as to restore the sensitivity of SCF to resistant strains and play the synergistic effect of combined use of drugs [33].

\section{CONCLUSION}

Baicalin combined with cefoperazone sulbactam sodium has a certain synergistic antibacterial or bactericidal effect on drug-resistant strains of Pseudomonas aeruginosa. It can provide a new way and experimental reference for the clinical treatment of drug-resistant bacteria and the development of new drug-resistant bacteria. At the same time, it also has a certain practical guiding significance for the prevention and treatment of PAE drug resistance.

\section{LIMITATIONS OF THE STUDY}

The samples of this study are from patients in the District of this city, which has regional differences. In the next study, it is planned to collect samples from more regions and extract more strains of Pseudomonas aeruginosa for research to reduce regional differences.

\section{ACKNOWLEDGEMENTS}

During the process of this topic research, we got much help from many departments and individuals, and other personnel not involved in this project research. All of them offered a great support and help in this research. Now here, all of members in this research show our deepest appreciation to them, and wish them good health and everything goes well.

\section{FUND PROJECT}

This study is supported by the Scientific Research Project of Guangxi Zhuang Autonomous Region Health Commission (Z20190417).

\section{CONFLICTS OF INTEREST}

The authors declare no conflicts of interest regarding the publication of this paper.

\section{REFERENCES}

1. Yu, Y., Shao, X.S., Yang, W.P., et al. (2018) Clinical Distribution and Drug Resistance of Pseudomonas aeruginosa in a Tertiary Hospital. Chinese Journal of Disinfection, 35, 63-66.

2. Liu, Y., Wang, M.Q., Zhu, B.T., et al. (2019) Drug Resistance Analysis and Homology Study of Pseudomonas aeruginosa in Packaged Drinking Water. China Brewing, 6, 132-135.

3. Wagner, S., Sommer, R., Hinsberger, S., et al. (2016) Novel Strategies for the Treatment of Pseudomonas aeru- 
ginosa Infections. Journal of Medicinal Chemistry, 59, 5929-5969.

https://doi.org/10.1021/acs.jmedchem.5b01698

4. Valentini, M. and Filloux, A. (2016) Biofilms and c-di-GMP Signaling: Lessons from Pseudomonas aeruginosa and Other Bacteria. Journal of Biological Chemistry, 291, 12547-12555. https://doi.org/10.1074/jbc.R115.711507

5. Weinstein, M.P. and Lewis, J.S. (2020) The Clinical and Laboratory Standards Institute (CLSI) Subcommittee on Antimicrobial Susceptibility Testing: Background, Organization, Functions, and Processes. Journal of Clinical Microbiology, 58, e01864-19. https://doi.org/10.1128/JCM.01864-19

6. Shang, H., Wang, Y.S. and Shen, Z.Y. (2015) National Clinical Testing Operational Regulations. People's Health Publishing House, Beijing, 11-50.

7. Humphries, R., Bobenchik, A.M., Hindler, J.A. and Schuetz, A.N. (2021) Overview of Changes to the Clinical and Laboratory Standards Institute Performance Standards for Antimicrobial Susceptibility Testing, M100, 31st Edition. Journal of Clinical Microbiology, 59, e0021321. https://doi.org/10.1128/JCM.00213-21

8. Zhang, P., Guo, Q., Wei, Z., et al. (2021) Baicalin Represses Type Three Secretion System of Pseudomonas aeruginosa through PQS System. Molecules, 26, 1497. https://doi.org/10.3390/molecules26061497

9. Luo, J., Dong, B., Wang, K., et al. (2017) Baicalin Inhibits Biofilm Formation, Attenuates the Quorum Sensing-Controlled Virulence and Enhances Pseudomonas aeruginosa Clearance in a Mouse Peritoneal Implant Infection Model. PLoS ONE, 12, e0176883. https://doi.org/10.1371/journal.pone.0176883

10. Zhou, X.L., Sun, X.X., Yan, J., et al. (2005) Antibacterial Activity of Cefoperazone Sulbactam against Pseudomonas aeruginosa. Chinese Journal of Anti-Infective Chemotherapy, No. 1, 44-45.

11. Cui, Y.X. (2003) Analysis of Drug Resistance of Cefoperazone/Sulbactam and Other Antibiotics to Various Types of Pseudomonas aeruginosa. Henan Journal of Oncology, No. 4, 300-301.

12. Dong, B.Y. (2016) Baicalein Inhibits the Motility of Pseudomonas aeruginosa and the Synergistic Effect of Cefoperazone/Sulbactam on Its Biofilm in Vitro. Guangxi Medical University, Nanning.

13. Dong, B.Y., Chen, Y.Q., Kong, J.L., et al. (2016) In Vitro Study of Baicalin Combined with Cefoperazone/ Sulbactam on the Destruction of Pseudomonas aeruginosa Biofilm. Chinese Journal of Modern Medicine, 18, $1-4$.

14. Bayot, M.L. and Bragg, B.N. (2020) Antimicrobial Susceptibility Testing.

15. Katawa, G., Agbemanyole, K.A., Tchopba, C.N., et al. (2021) Antimicrobial Susceptibility Testing.

16. Arakawa, Y., et al. (2000) Convenient Test for Screening Metallo- $\beta$-Lactamase-Producing Gram-Negative Bacteria by Using Thiol Compounds. Journal of Clinical Microbiology, 38, 40-43.

https://doi.org/10.1128/JCM.38.1.40-43.2000

17. Li, S.H., Li, D., Yu, F.M., et al. (2017) In Vitro Antibacterial Activity of Cefoperazone/Sulbactam against Acinetobacter baumannii and Klebsiella pneumoniae. Chinese Journal of Hospital Infectious Diseases, 27, 1452-1455.

18. Gong, M.L., Li, X.X., Zhou, Y., et al. (2016) Inhibition of Carbapenem-Resistant Acinetobacter baumannii in Combination with Etimicin and Cefoperazone/Sulbactam and Piperacillin/Tazobactam in Vitro Role. Journal of PLA Medical College, 37, 876-878.

19. Xu, A.X., Zeng, X., Hu, Y., et al. (2019) In Vitro Antibacterial Effects of Psyllium Extract and Cefoperazone Sodium/Sulbactam Sodium on ESBLs-Producing Escherichia coli. Jiangxi Medicine, 54, 1034-1036.

20. Wang, Q.M., Lu, D.Q., Peng, M., et al. (2019) Clinical Distribution and Drug Resistance Analysis of 2279 Strains of Pseudomonas aeruginosa for 5 Consecutive Years. Laboratory Medicine and Clinic, 16, 3437-3440.

21. Hoffman, L., D’Argenio, D., Maccoss, M., et al. (2005) Aminoglycoside Antibiotics Induce Bacterial Biofilm Formation. Nature, 436, 1171-1175. https://doi.org/10.1038/nature03912 
22. Huang, X.H., Gao, G.Y. and Huang, Z.A. (2018) In Vitro Antibacterial Effects of 12 Traditional Chinese Medicines on 20 Strains of Extensively Drug-Resistant Pseudomonas aeruginosa. China Practical Medicine, 13, 196-198.

23. Zhou, X. and Zhou, B.J. (2017) In Vitro Antibacterial Activity of 113 Traditional Chinese Medicine Decoctions against Pseudomonas aeruginosa. Chinese Medicine Forum, No. 4, 59-61.

24. Xie, L.L., Zhou, M., Chen, Y.C., et al. (2010) Study on the Inhibition of Pseudomonas aeruginosa Biofilm Formation by Baicalin and Baicalein. China Pharmacy, 21, 3651-3653.

25. Jian, L.Y., He, X.J. and Yu, Y. (2012) Effects of Baicalin Combined with Levofloxacin on Pseudomonas aeruginosa Biofilm and Related Mechanisms. Chinese Journal of Hospital Pharmacy, 32, 1097-1099.

26. Cen, Y.L., Li, Y.N., Kong, J.L., et al. (2017) In Vivo Effects of Baicalin Combined with Meropenem on the Early Biofilm of Pseudomonas aeruginosa in the Peritoneal Cavity of Mice. Chinese Journal of Hospital Infection, 27, 39-42.

27. Lu, D.Y. and Lu, T.R. (2020) Drug Sensitivity Testing, a Unique Drug Selection Strategy. Advances in Biomarker Sciences and Technology, 2, 59-66. https://doi.org/10.1016/j.abst.2020.11.001

28. Yin, D., Wu, S., Yang, Y., Shi, Q., Dong, D., Zhu, D. and Hu, F. (2019) Results from the China Antimicrobial Surveillance Network (CHINET) in 2017 of the in Vitro Activities of Ceftazidime-Avibactam and Ceftolozane-Tazobactam against Clinical Isolates of Enterobacteriaceae and Pseudomonas aeruginosa. Antimicrobial Agents and Chemotherapy, 63, e02431-18. https://doi.org/10.1128/AAC.02431-18

29. Olivares, P.J., Alvarez Ortega, C. and Luis, M.J. (2014) Metabolic Compensation of Fitness Costs Associated with Over-Expression of the Multidrug Efflux Pump MexEF-OprN in Pseudomonas aeruginosa. Antimicrobial Agents and Chemotherapy, 58, 3904-3913. https://doi.org/10.1128/AAC.00121-14

30. Topka-Bielecka, G., Dydecka, A., Necel, A., et al. (2021) Bacteriophage-Derived Depolymerases against Bacterial Biofilm. Antibiotics, 10, 175. https://doi.org/10.3390/antibiotics10020175

31. Xu, H.Y., Wang, S.F., Wang, L. and Wang, H. (2013) Study on the Antibacterial Effect of Shuanghuanglian Oral Liquid Combined with Gentamicin in Vitro. Medicine Herald, 32, 19-22.

32. Wang, L.J., Zeng, J.M., Lu, Y., Lan, K., Luo, Q., Lin, D.L., Zhang, W.Z. and Chen, C. (2017) Study on the Effect of Traditional Chinese Medicine on the Efflux Pump of Multidrug-Resistant Pseudomonas aeruginosa. Journal of Molecular Diagnosis and Therapy, 9, 401-407.

33. Ionescu, C.M., Ghita, M., Copot, D., et al. (2020) A Minimal PKPD Interaction Model for Evaluating Synergy Effects of Combined NSCLC Therapies. Journal of Clinical Medicine, 9, 1832.

https://doi.org/10.3390/jcm9061832 